\title{
Ultrafast optical rotations of electron spins in
} \section{quantum dots}

\author{
A. Greilich ${ }^{1 \star}$, Sophia E. Economou ${ }^{2 \star}$, S. Spatzek ${ }^{1}$, D. R. Yakovlev ${ }^{1,3}$, D. Reuter ${ }^{4}$, A. D. Wieck ${ }^{4}$ \\ T. L. Reinecke ${ }^{2}$ and M. Bayer ${ }^{1}$
}

Coherent manipulation of quantum bits (qubits) on timescales much shorter than the coherence time ${ }^{1,2}$ is a key prerequisite for quantum information processing. Electron spins in quantum dots are particularly attractive for implementations of qubits, and efficient optical methods for initialization and readout of spins have been developed in recent years ${ }^{3,4}$. Spin coherence times in the microsecond range have been demonstrated ${ }^{5}$. Therefore, spin control by picosecond optical pulses would be highly desirable so that a large number of spin rotations could be carried out while coherence is maintained. A major remaining challenge is demonstration of such rotations with high fidelity. Here, we use an ensemble of quantum-dot electron spins focused into a small number of precession modes about a magnetic field by periodic optical pumping. We demonstrate ultrafast optical rotations of spins about arbitrary axes on a picosecond timescale using laser pulses as control fields.

Spin rotations have been obtained for gated GaAs transport quantum dots using radiofrequency fields. But such manipulations are slow, with times comparable to the spin coherence time ${ }^{6}$. Fast optical rotation operations on spins in quantum dots are being sought actively, but progress so far has been limited. In an all-optical experiment on shallow interface-fluctuation quantum dots, weak confinement precluded full unitary spin rotations ${ }^{7}$. Evidence for spin rotations was reported for a single GaAs interface-fluctuation quantum dot using very intense optical excitation needed for spins to be rotated by precession about the effective laser magnetic field ${ }^{8}$. Very recently, convincing rotations in a single InAs self-organized quantum dot were reported ${ }^{9}$. This work on single quantum dots $\mathrm{s}^{8,9}$ used pulses far from resonance, which could lead to excitation of other spins. This could inhibit the use of spins with separations less than the laser spot size for two-quantum-bit entanglement. In general, optical coupling to a spin in a single quantum dot is weak, and thus spin rotations on single quantum dots give weak responses. This generally necessitates many optical pulses in time to obtain sufficient fidelity, and it can lead to extra dephasing.

An ensemble of quantum dots has the advantage of increasing the optical coupling and can decrease the loss of information from the loss of a few spins. However, ensemble approaches typically are hampered by the inhomogeneities in quantum dot properties, particularly in the spin-splitting $g$-factor, and lead to dephasing of the spin precession about a magnetic field ${ }^{10,11}$. Recently we have demonstrated techniques for eliminating part of the inhomogeneity in the electron spin precession in ensembles of singly charged quantum dots ${ }^{12}$. We have found that at low magnetic fields the spin ensemble can be put into a Zeeman spectrum $^{13}$ that is very close to a single precession mode. This is the system that we study a

C

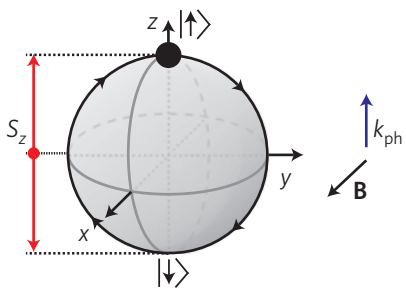

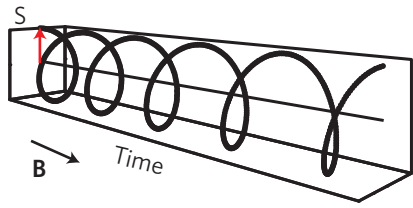

b $\sigma+$ pump with intensity $\pi$

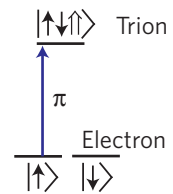

d $\sigma+$ control with intensity $2 \pi$

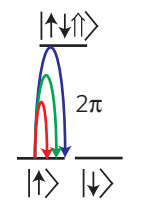

Figure 1 | Optical methods to control spins. a, Larmor precession of a spin $\mathrm{S}$ about the magnetic field. $\mathbf{b}$, Spin polarization is generated by laser pulses resonant with the trion (two electrons and one hole) transition. Excitation with area $\Theta=\pi$ pulses is most efficient to drive the system to the trion state and to generate electron spin coherence after trion spontaneous decay $^{23}$. c, Precession of the spin around the magnetic field is represented on a Bloch sphere by the black trajectory with arrows. The measured ellipticity is proportional to the projection of electron spin polarization on the $z$ axis $\left(S_{z}\right) \cdot k_{\text {ph }}$ is the wave vector of the pump light generating electron polarization along the $z$ axis. $\mathbf{d}, A 2 \pi$ control pulse does not change the population of the electron and trion states. It provides rotation of the spins around the $z$ axis with the angle depending on laser detuning from the trion resonance.

here. Measurements of such systems demonstrate homogeneous spin dephasing times to be at least $3 \mu$ s (ref. 5). Thus, for optical control on the picosecond timescale, about $10^{6}$ operations could be carried out during the coherence time.

Our samples consist of arrays of self-organized (In,Ga)As quantum dots, each containing on average a single electron ${ }^{14}$ (see Supplementary Information). The spins are initialized in the $z$ direction by periodic optical laser pulses. These spins are polarized along the light propagation direction, which is parallel to the quantum-dot-growth direction. A magnetic field $\mathbf{B}$ is applied along the $x$ axis (Voigt geometry). Instantaneous orientation of the spins along the optical axis is obtained through optical pumping by pulses with area $\Theta=\int \mathrm{d} t \mathbf{d} \cdot \mathbf{E} / \hbar$ resonant with the trion transitions (Fig. 1b). E is the electric field of the laser, which excites an inhomogeneous distribution of quantum dots with

${ }^{1}$ Experimentelle Physik 2, Technische Universität Dortmund, 44221 Dortmund, Germany, ${ }^{2}$ Naval Research Laboratory, Washington, DC 20375, USA, ${ }^{3}$ A. F. loffe Physico-Technical Institute, Russian Academy of Sciences, 194021, St. Petersburg, Russia, ${ }^{4}$ Angewandte Festkörperphysik, Ruhr-Universität Bochum, 44780 Bochum, Germany. *e-mail: alex.greilich@udo.edu; economou@bloch.nrl.navy.mil. 

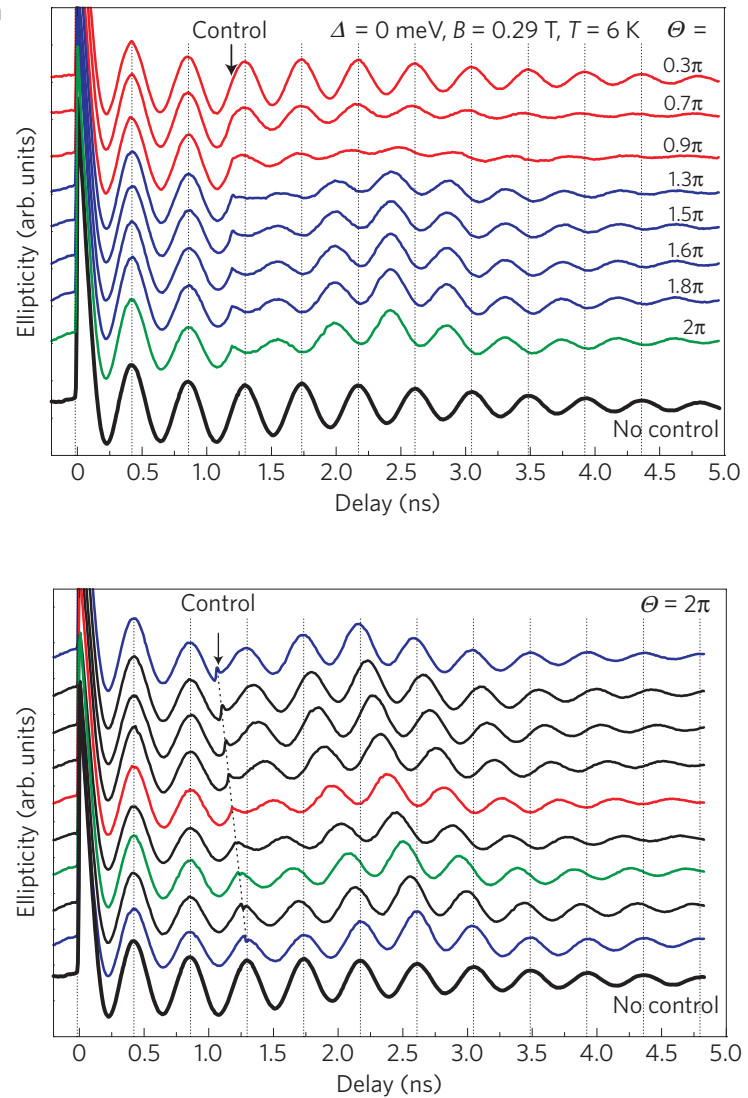

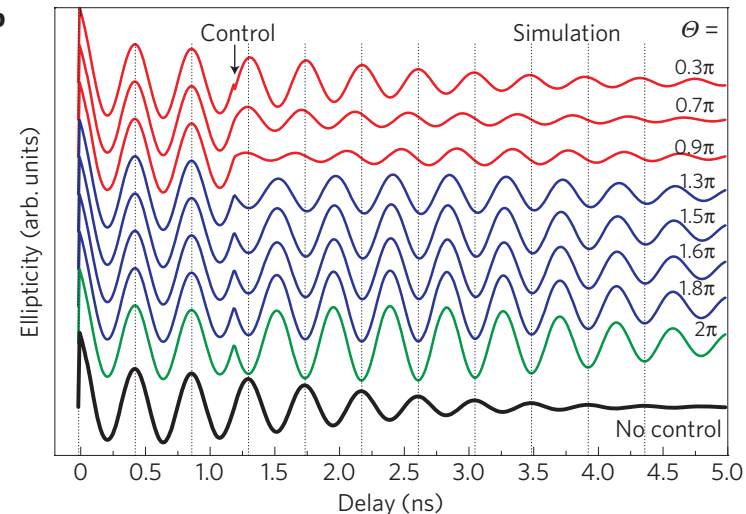

d

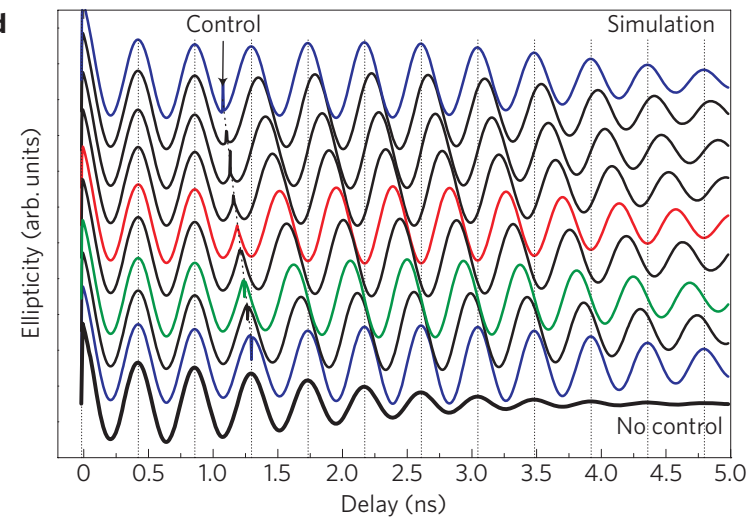

e

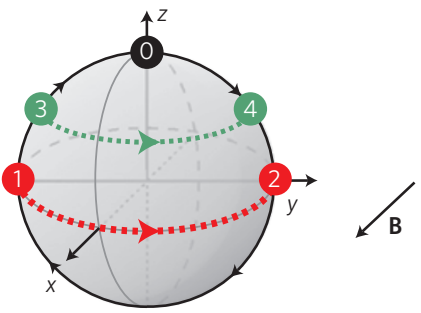

Control $\Theta=2 \pi, \varphi=\pi$

Figure 2 | Single-pulse spin control. a, Measurements of ellipticity of the total spin of an ensemble versus delay time between the pump and probe. The pump and probe energies are degenerate, and the control energy is the same as that of the pump. The bottom (black) trace is without a control laser. The control hits the sample $1.2 \mathrm{~ns}$ after the pump, when the spin vector is along the $-y$ direction. The control area $\Theta$ is varied to identify the $2 \pi$ pulse through a phase shift of $\pi$ of the spin relative to no control. $\mathbf{b}$, Simulations corresponding to the traces in a (see Supplementary Information). In the simulations, all quantum dots have the same pulse intensity. Reduction in the amplitude after the control in the experiment is attributed primarily to inhomogeneities in the dipole in different quantum dots, which causes part of them to see a pulse area other than $\Theta=2 \pi$. c, Ellipticity measurements for control area $\Theta=2 \pi$ versus pump-probe delay. The bottom (black) trace is without a control laser. Other traces are with the control at varying delays after the pump.

d, Simulations corresponding to c. e, The effects of the control delay sketched on the Bloch sphere. The spin is oriented along the $z$ axis by the pump, shown by the black dot marked with zero. It precesses about the magnetic field until it is hit by the control, which induces a $\pi$ rotation about $z$. Then it continues its precession. The two trajectories have colours corresponding to the green and red traces of $\mathbf{c}$.

varying dipole matrix elements $\mathbf{d}$ and energies of the trion optical transition. In the present experiments, the spectroscopic responses are obtained from an average over many dots. The pulse intensities are adjusted to achieve the desired average $\Theta$ in the ensemble (see the Methods section).

The precession of spins (Fig. 1a) is measured by the ellipticity of a probe laser and is proportional to the spin polarization along the optical axis $z$. The spin vector oscillates about $\mathbf{B}$, which is represented on the Bloch sphere by a precession in the $y-z$ plane (Fig. 1c). We use ultrafast 'control' laser pulses to induce rotations of the spins. The axis of the spin rotation is given by the polarization of the control laser, and the rotation angle is determined by the photon energy detuning $\Delta$ of the control pulse from the optical resonance (Fig. 1d). By using $\Theta=2 \pi$ pulses and varying their detuning, we implement spin rotations by angles from $0.2 \pi$ to $\pi$ and find that the detuning dependence of the angle of rotation agrees well with what we expect from theory ${ }^{15,16}$. By combining two spin rotations by angles of $\pi / 2$ with precession-induced rotation, we obtain a composite rotation about the $y$ axis. This can give spin rotations about arbitrary angles. For the first time for optically controlled spins, we see spin echoes and extension of the dephasing time of the spins $T_{2}^{*}$. This opens opportunities for ultrafast decoupling operations to provide increased single spin coherence times $T_{2}$ (refs 17,18).

We used two synchronized Ti:sapphire lasers each emitting pulses of 1.5-ps duration with a separation of $T_{R}=13.2 \mathrm{~ns}$. 

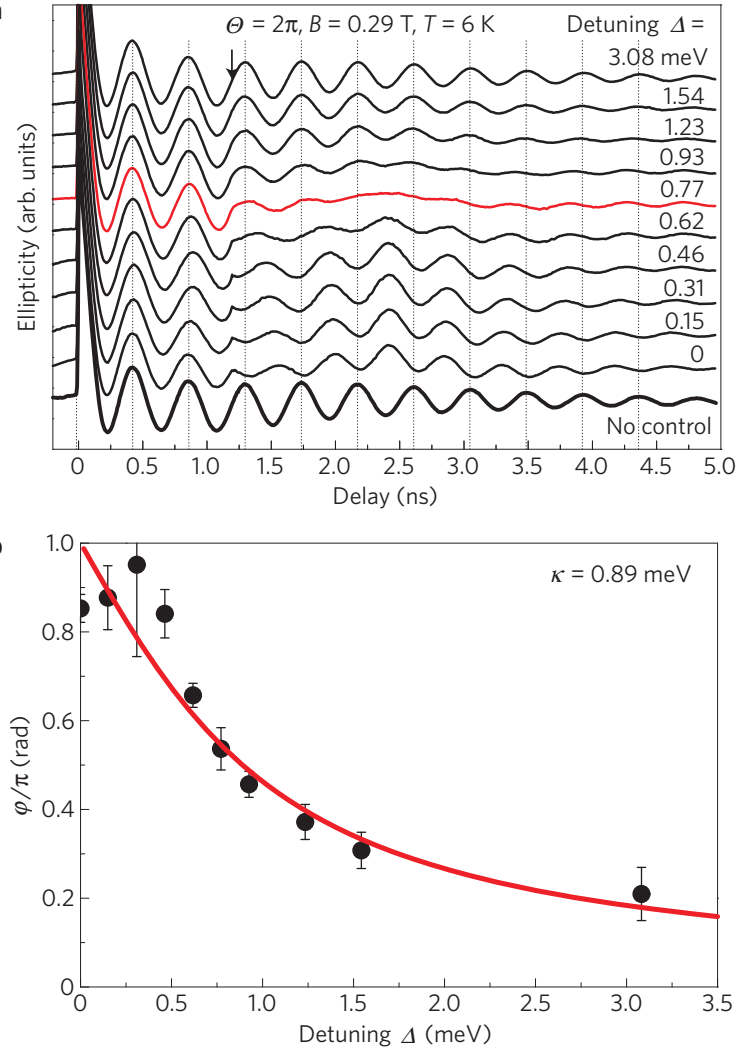

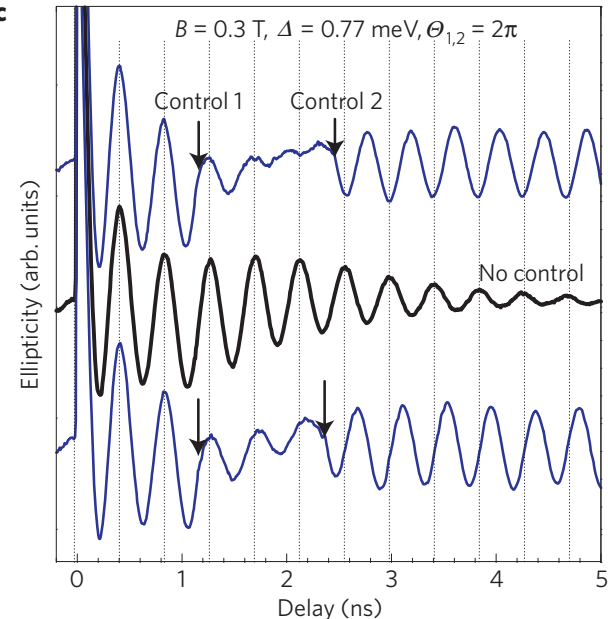

d

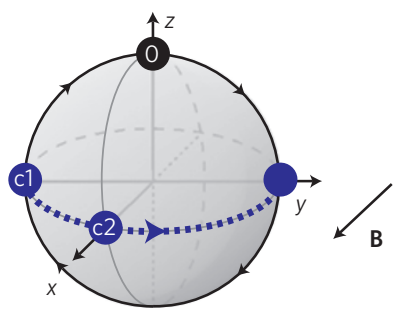

Figure 3 | Two-pulse control. a, Measurements of ellipticity for varying detunings between control and pump energies. Control pulse intensities are $\Theta=2 \pi$, and the delays between the pump and control pulses were chosen so that the spin is along the $-y$ direction when the control is applied. $\mathbf{b}$, Symbols give the dependence of rotation angle on the detuning between the control and pump from the data in $\mathbf{a}$. The red line gives the theoretical result $\varphi=2 \arctan (\kappa / \Delta)$ from refs 15,16 . c, Ellipticity measurements with two control pulses with varying temporal separations between them. The two control pulses have an area of $2 \pi$ and the same energy. Their detuning from the pump photon energy is $0.77 \mathrm{meV}$ so that each of them would give a $\pi / 2$ rotation about the $z$ axis, as in the single-control case. Together they have the effect of a single $\pi$ rotation about the $z$ axis. $\mathbf{d}$, Bloch sphere depiction of the two-control experiment. The points marked c1 and c2 are where the first and second control pulses hit the spin, respectively.

The pump and probe beams originate from the same laser (see Supplementary Information). The ellipticity with no control laser is shown by the bottom (black) trace in Fig. 2a versus pumpprobe delay. The precession of the spins exhibits a weak dephasing arising from the distribution of precession frequencies around the main mode $N \cdot 2 \pi / T_{\mathrm{R}}$ with $N=30$ at $B=0.29 \mathrm{~T}$ (ref. 5 ).

The control laser is tuned in the vicinity of the trion resonance. The goal is to manipulate the spin but to avoid generating new spin polarization. Unlike the case of radiofrequency control ${ }^{6}$, these optical pulses result in the spin state acquiring a trion component and partially exiting the qubit subspace. To return the state to the qubit subspace, the control must be unitary within that subspace. This is done by using a control pulse area of $2 \pi$, which moves the (optical) polarization through a full rotation (a Rabi flop, see Fig. 1d) to the optically excited state and back to the ground state. Then, a phase $\varphi$ is induced to the ground state. Owing to selection rules, this is a relative phase between the spin states pointing along $+z$ and $-z$, and it is equivalent to a rotation about the $z$ axis by angle $\varphi$ (see Supplementary Information).

To find the pulse of area $\Theta=2 \pi$, we varied the control intensity (Fig. 2a). Figure $2 \mathrm{~b}$ shows corresponding simulations, made by solving the complete dynamics of the three-level system of Fig. 1b (see Supplementary Information). Pulses with area $\Theta<\pi$ move some of the state to the excited trion level without returning it to the spin. Then the spin amplitude is reduced while its phase remains unchanged (top three curves in Fig. 2a). For $\Theta>\pi$, part of the state is returned to the spin subspace, so that the phase of the spin changes but the amplitude is reduced
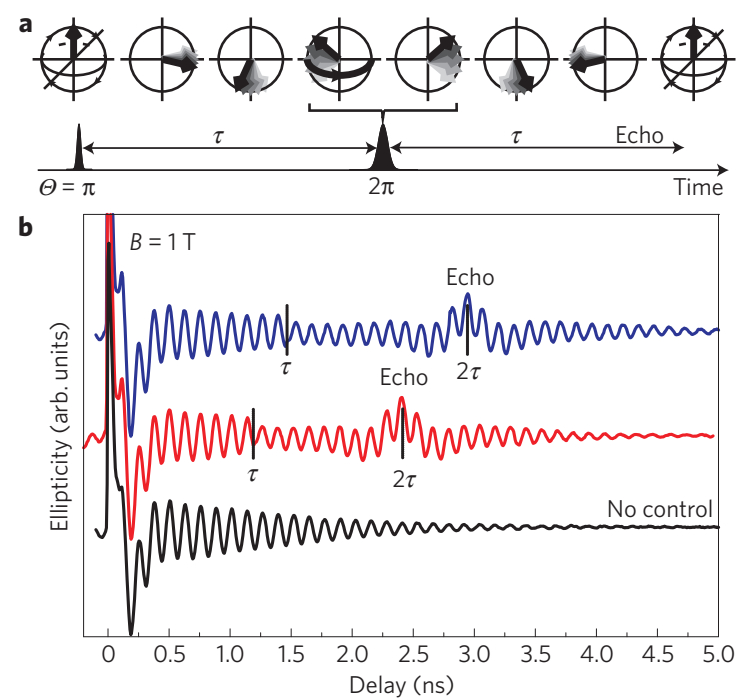

Figure 4 | Spin echoes. a, Schematic diagram of spin echo formation versus time in an optically controlled inhomogeneous spin ensemble. The spins are initialized along $z$ by a pulse of area $\pi$ after which they precess about the external magnetic field with different frequencies. At time $\tau$, a control pulse of area $\Theta=2 \pi$ and zero detuning rotates the spins about the $z$ axis by an angle $\pi$. After $2 \tau$, the spins come into phase again and an echo builds up. $\mathbf{b}$, Experimental demonstration of a spin echo for two time delays of 1.47 and 1.20 ns between pump and control pulses. 
compared with the case without control (blue curves in Fig. 2a). The green trace in Fig. $2 a$ is for the $\Theta=2 \pi$ control pulse, and it has the maximum amplitude among cases with control pulses. Its amplitude is reduced from the no control case owing to inhomogeneities in dipoles.

Next we fix $\Theta=2 \pi$ and vary the delay $\tau$ between the pump and control. This enables the spin to precess in the $y-z$ plane for a time $\tau$ (Fig. 2c). The blue traces in Fig. $2 \mathrm{c}$ correspond to applying the control pulse when the spin points in the $z$ direction, in which case it has basically no effect on the spin. For all other spin orientations, the control pulse gives a $\pi$ rotation about the $z$ axis, after which the spins continue to precess. Figure $2 \mathrm{e}$ shows control-induced rotations for two orientations. For the red trace, the control pulse was applied when the spin points in the $-y$ direction. After the control, it is completely out of phase with the reference trace.

Spin rotations of varying angles can be obtained by changing the detuning of the control pulse from the optical resonance while keeping its area at $2 \pi$. Figure $3 a$ gives a series of traces with the detunings between 0 and $3.08 \mathrm{meV}$. We choose the arrival time of the control so that the net spin polarization is along $-y$, in the equatorial plane of the Bloch sphere. Then the effect of rotation by the control becomes most prominent in the phase and amplitude of the ellipticity signal. The angle of spin rotation $\varphi$ decreases from $\pi$ to nearly zero as the detuning is increased from zero to $3.08 \mathrm{meV}$ (Fig. 3a). For angles of rotation $\pi / 2<\varphi<\pi$, the spin polarization immediately after the control has a component along $+y$ and a non-zero component along the magnetic field. Then the phase of the signal is opposite to that of the reference (Fig. 3a for detunings $<0.77 \mathrm{meV}$ ). For $\varphi<\pi / 2$, the $y$ component is still negative immediately after the control, and the oscillations are in phase with the reference. The angles of rotation are obtained from the ratio of the (signed) amplitude over the amplitude of the reference at the maximum signal at $2.4 \mathrm{~ns}$. It is shown as a function of detuning in Fig. 3b. This angle is in excellent agreement with the theoretical expression, which is derived analytically for pulses of hyperbolic secant temporal profile $\operatorname{sech}(\kappa \cdot t)$ and gives $\varphi=2 \arctan (\kappa / \Delta)$, where $\kappa$ is the bandwidth of the pulse $e^{15,16}$.

The two experiments in blue in Fig. $3 \mathrm{c}$ demonstrate further the effects of spin rotations by angle $\pi / 2$ obtained by using the detuning $\Delta=0.77 \mathrm{meV}$. For each, there are two controls giving two $\pi / 2$ rotations, with varying delays between them. In both, the first control is applied when the spin is in the $-y$ direction, rotating the spin into the $+x$ direction, and the second control rotates the spin back into the $y-z$ plane in the $+y$ direction. In the lower case, the second rotation is applied when the reference is pointing in the $-z$ direction, which results in a phase difference of $\pi / 2$ between the two applied controls and the reference (Fig. 3c). In the upper case, the second control is applied when the reference spin is in the $-y$ direction, resulting in a phase difference of $\pi$ between the two-control case and the reference.

Spin echoes are seen in Figs 2 and 3 as an increase of signal amplitude around time delays of $2 \tau$. They originate from inhomogeneities in the spin precession frequencies. A $\pi$ rotation at $\tau$ on a set of inhomogeneously precessing spins that were aligned at time zero causes the slower spins to advance ahead of the faster ones along their precession direction (Fig. 4a). Then the spins refocus at $2 \tau$, a phenomenon known from NMR as spin echo $^{19}$ (spins echoes for nuclear spin ensembles have been seen in single gated quantum dots in ref. 20). The observation of electron-spin echoes in our experiments demonstrates the robustness of the spin manipulations carried out here. This spin echo is seen in Fig. 2 a,c and also for a rotation by $\varphi>\pi / 2$ (for $0 \leqslant \Delta \leqslant 0.62 \mathrm{meV}$ ) in Fig. 3a. However, no echo is seen for $\varphi<\pi / 2(\Delta>0.8 \mathrm{meV})$. This is because in that case the slower spins are further delayed instead of being moved ahead of the fast ones. The echo signal became more pronounced at a stronger magnetic field of $1 \mathrm{~T}$, as can be seen in Fig. $4 \mathrm{~b}$ for zero detuning of the $2 \pi$ control pulse and for two different delays. Thus, we are able to increase the dephasing time of the ensemble, $T_{2}^{*}$, as seen by comparing the amplitude after $2 \tau$ of the rotated spins with the reference. In principle, these $\pi$ rotations can also extend the $T_{2}$ time of the individual spins by decoupling them ${ }^{17}$ or disentangling them ${ }^{21,22}$ from the nuclei.

This work demonstrates robust, optically controlled single spin rotation gates on the picosecond timescale in quantum-dot ensembles. These gates could provide the basis for single-qubit operations, and thus are an important step forward in spin-based quantum-dot implementations for quantum information.

The present quantum-dot experiments were done on ensembles, and they give information about the control of spins at the singledot level. The fidelity of the coherent operations could be affected by dephasing in the quantum dots owing, for example, to decay of optically inactive states or many-body effects, as well as to the effects of inhomogeneities in dipoles or transition energies. For applications in quantum information, these matters would need to be assessed by, for example, full quantum-state tomography, which is beyond the scope of this work.

\section{Methods}

Two synchronized Ti:sapphire lasers provide trains of 1.5-ps-long pulses at $75.6 \mathrm{MHz}$ repetition frequency, which corresponds to $13.2 \mathrm{~ns}$ of pulse separation. The first laser is split to give the pump and probe laser beams at the same energy. The second laser gives the extra control beams, and its energy can be tuned independently relative to the energy of the pump and probe. We use a phase-sensitive lock-in technique for time-resolved ellipticity measurements to trace the spin coherence excited by the circularly polarized pump. The time delay between the pump and probe is obtained by a mechanical delay line. An extra delay line was used to vary the delay between the pump and control pulses.

The generation of spin coherence is most efficient for a $\Theta=\pi$ pump pulse area, whereas the control pulse should have an area of $2 \pi$. Owing to inhomogeneities of the dipole transition matrix element in the ensemble, the pulse area will not be uniform for all of the dots. For the ones that see a pulse area other than $2 \pi$, new spin coherence will be generated. To avoid picking up this contribution, we modulate only the pump beam with a photo-elastic modulator. As can be seen from Supplementary Fig. S1, this extra polarization is minimal, which enables us to use the label of $\Theta=2 \pi$ to a very good approximation when discussing the dynamics of the ensemble. Moreover, the control beam diameter was chosen to be larger than those of the pump and probe to achieve a high homogeneity of the control intensity over the spin coherence generated by the pump.

Received 5 December 2008; accepted 12 February 2009; published online 22 March 2009

\section{References}

1. Burkard, G., Engel, H. A. \& Loss, D. Spintronics and quantum dots for quantum computing and quantum communication. Prog. Phys. 48, 965-986 (2000).

2. Pryor, C. E. \& Flatté, M. E. Predicted ultrafast single-qubit operations in semiconductor quantum dots. Appl. Phys. Lett. 88, 233108 (2006).

3. Atatüre, M. et al. Quantum-dot spin-state preparation with near-unity fidelity. Science 312, 551-553 (2006).

4. Xu, X. et al. Fast spin state initialization in a singly charged InAs/GaAs quantum dot by optical cooling. Phys. Rev. Lett. 99, 097401 (2007).

5. Greilich, A. et al. Mode locking of electron spin coherences in singly charged quantum dots. Science 313, 341-345 (2006).

6. Koppens, F. H. L. et al. Driven coherent oscillations of a single electron spin in a quantum dot. Nature 442, 766-771 (2006).

7. Wu, Y. et al. Selective optical control of electron spin coherence in singly charged GaAs/AlGaAs quantum dots. Phys. Rev. Lett. 99, 097402 (2007).

8. Berezovsky, J., Mikkelsen, M. H., Stoltz, N. G., Coldren, L. A. \& Awschalom, D. D. Picosecond coherent optical manipulation of a single electron spin in a quantum dot. Science 320, 349-352 (2008).

9. Press, D., Ladd, T. D., Zhang, B. \& Yamamoto, Y. Complete quantum control of a single quantum dot spin using ultrafast optical pulses. Nature 456, 218-221 (2008).

10. Khaetskii, A. V., Loss, D. \& Glazman, L. Electron spin decoherence in quantum dots due to interaction with nuclei. Phys. Rev. Lett. 88, 186802 (2002).

11. Merkulov, I. A., Efros, A. L. \& Rosen, M. Electron spin relaxation by nuclei in semiconductor quantum dots. Phys. Rev. B 65, 205309 (2002). 
12. Greilich, A. et al. Nuclei-induced frequency focusing of electron spin coherence. Science 317, 1896-1899 (2007).

13. Greilich, A. et al. Collective single mode precession of electron spins in a quantum dot ensemble. Preprint at $<$ http://arxiv.org/abs/0811.1783 $>$ (2008).

14. Yugova, I. A. et al. Exciton fine structure in InGaAs/GaAs quantum dots revisited by pump-probe Faraday rotation. Phys. Rev. B 75, 195325 (2007).

15. Economou, S. E. \& Reinecke, T. L. Theory of fast optical spin rotation in a quantum dot based on geometric phases and trapped states. Phys. Rev. Lett. 99, 217401 (2007).

16. Economou, S. E., Sham, L. J., Wu, Y. \& Steel, D. G. Proposal for optical $\mathrm{U}(1)$ rotations of electron spin trapped in a quantum dot. Phys. Rev. B 74, 205415 (2006).

17. Viola, L. \& Lloyd, S. Dynamical suppression of decoherence in two-state quantum systems. Phys. Rev. A 58, 2733-2744 (1998).

18. Morton, J. J. L. et al. Bang-bang control of fullerene qubits using ultrafast phase gates. Nature Phys. 2, 40-43 (2006).

19. Hahn, F. Spin echoes. Phys. Rev. 80, 580-594 (1950).

20. Koppens, F. H. L., Nowack, K. C. \& Vandersypen, L. M. K. Spin echo of a single electron spin in a quantum dot. Phys. Rev. Lett. 100, 236802 (2008).
21. Yao, W., Liu, R.-B. \& Sham, L. J. Restoring coherence lost to a slow interacting mesoscopic spin bath. Phys. Rev. Lett. 98, 077602 (2007).

22. Witzel, W. M. \& Das Sarma, S. Multiple-pulse coherence enhancement of solid state spin qubits. Phys. Rev. Lett. 98, 077601 (2007).

23. Shabaev, A., Efros, A. L., Gammon, D. \& Merkulov, I. A. Optical readout and initialization of an electron spin in a single quantum dot. Phys. Rev. B 68, 201305(R) (2003)

\section{Acknowledgements}

This work was supported by the Bundesministerium für Bildung and Forschung program 'nanoquit', the Office of Naval Research and the Deutsche Forschungsgemeinschaft. S.E.E. was an NRC/NRL Research Associate during this work.

\section{Additional information}

Supplementary Information accompanies this paper on www.nature.com/naturephysics. Reprints and permissions information is available online at http://npg.nature.com/ reprintsandpermissions. Correspondence and requests for materials should be addressed to A.G. or S.E.E. 